\title{
La division du travail de formation dans les formations professionnelles initiales: causes sociales et conséquences didactiques
}

\section{Laurent Veillard}

Depuis la période des révolutions industrielles et politiques, de nombreux états ont élaboré des systèmes de formation professionnelle initiale avec des structures spécifiques. Dans cet article, on analyse les discours des grandes organisations internationales qui recommandent aux états de réformer leur système de formation autour de quelques principes communs. Puis on s'appuie sur des théories de la différenciation en sociologie pour montrer que les réformes menées, souvent en cohérence avec ces recommandations, répondent à des processus de différenciation sociale profonds dans nos sociétés, conduisant à une division du travail de formation croissante au sein des cursus formatifs. Enfin, on examine les conséquences de ces transformations sur les parcours d'apprentissage des élèves.

\section{Introduction}

Depuis la période des révolutions industrielles et politiques, de nombreux états nationaux ont élaboré des systèmes de formation professionnelle initiale avec des structures spécifiques pour tenter de répondre aux besoins de main d'œuvre suscités par le développement économique et l'industrialisation du pays. Plusieurs études ont montré que ces spécificités étaient liées à des évolutions historiques propres à chaque espace national sur les plans politique, économique et sociétal (Billett, 2011; Evans, 2007; Greinert, 2004, 2005; Hyslop-Margison, 2000; Pelpel \& Troger, 1993). Les systèmes mis en place en France et en Allemagne illustrent bien ces spécificités.

En France, le choix d'une scolarisation forte de la formation professionnelle renvoie à un compromis lentement élaboré entre la fin du $19^{\mathrm{e}}$ et la première moitié du $20^{\mathrm{e}}$ siècle entre le grand patronat, les hauts-fonctionnaires des ministères de l'éducation et du commerce et de l'industrie, rejoints plus tardivement par les syndicats de salariés (Pelpel \& Troger, 1993). Ce compromis visait à rattraper un retard industriel et économique important, notamment vis-à-vis du voisin et rival anglais. Pour ce faire, bonne nombre d'élites françaises jugeaient 
les pratiques traditionnelles de travail inaptes à affronter les défis posés par les nouveaux outils ou méthodes de production (Greinert, 2004). Il fallait donc les remplacer par de nouvelles, basées sur la mise en application des savoirs scientifiques et techniques les plus récents. La rupture avec l'apprentissage corporatiste et la mise en place d'un système de formation séparé du monde du travail, ont paru la solution la plus pertinente pour y parvenir.

À l'inverse, en Allemagne, la foi dominante dans la tradition de métier, défendue par les classes moyennes et traditionnelles et relayée par le gouvernement de Bismarck à la fin du $19^{\mathrm{e}}$ siècle, a permis de maintenir les corporations et développer l'apprentissage au sein de celles-ci comme voie privilégiée de formation professionnelle. Il s'agissait de privilégier l'acquisition de gestes professionnels qui avaient fait leurs preuves dans des situations de travail complexes. Dans un tel cadre, les évolutions de la tradition n'étaient pas interdites, mais devaient faire l'objet d'un accord préalable entre professionnels. La formation par apprentissage constituait une bonne façon de perpétuer ou faire évoluer certaines pratiques, si on lui adjoignait des heures d'enseignement dans des centres de formation. On a ici les éléments de base du système dual mis en place.

Cependant, si les modèles nationaux issus de l'histoire gardent encore aujourd'hui des spécificités importantes, on peut faire l'hypothèse que l'on est entré dans une nouvelle période depuis une vingtaine d'années. Cette période semble caractérisée par des tentatives d'aller vers une plus grande convergence de ces systèmes nationaux, vers quelques principes pédagogiques ou didactiques communs. Cette hypothèse peut être posée si l'on considère que les processus de globalisation en cours ne concernent pas seulement la sphère des échanges économiques, mais aussi potentiellement d'autres sphères culturelles telles que les mondes de l'éducation et de la formation. Or, les grandes organisations internationales telles l'OCDE, l'Union Européenne, l'UNESCO, le Bureau International du Travail, la Banque Mondiale, souvent considérées comme des vecteurs ou des relais importants de ces processus de globalisation, produisent régulièrement et depuis plusieurs années des discours en matière d'éducation, en particulier en ce qui concerne la formation professionnelle initiale. Il est donc intéressant de se pencher sur ces discours pour les analyser et voir s'ils convergent.

Dans la première partie de cet article qui se situe à mi-chemin entre sociologie et didactique professionnelle, nous examinons plus en détail le contenu de ces discours pour mettre en évidence leurs invariants sur un plan particulier: celui des contenus de formation préconisés et des situations d'apprentissage à privilégier pour les transmettre. Puis, à partir de plusieurs travaux de recherche, nous étudions si ces préconisations rejoignent des évolutions en cours au sein des systèmes de formation professionnelle nationaux, en particulier dans l'Espace Européen. Dans la partie suivante, nous proposons de mobiliser des théories sociologiques sur les processus de différenciation et d'intégration sociales au sein des sociétés modernes pour approfondir les causes d'un processus de division du 
travail de formation croissant. En conclusion, nous discutons des conséquences potentielles que pose ce type d'évolution sur les plans pédagogique et didactique.

\section{Le discours des organisations internationales}

La plupart des textes produits depuis quelques années par les grandes institutions internationales constatent de fortes transformations du monde du travail, à la fois sur le plan des technologies, des organisations et des modes de travail, de la globalisation des échanges, de l'évolution rapide des marchés, et de la concurrence accrue entre pays du monde (Field, Hoeckel, Kis \& Kuczera, 2009; ILO, 2010; OCDE, 2015; Schleicher, 2006; The World Bank, 2002; UE, 2010; UNESCO-UNEVOC, 2015). De façon schématique, les organisations productives sont plus collaboratives, flexibles et réactives, pour répondre aux évolutions très rapides des marchés et des sociétés et pour faire face à des problèmes environnementaux et géopolitiques croissants. Les environnements professionnels sont aussi plus saturés de technologies numériques et les pratiques de travail requièrent à tous les niveaux des capacités d'analyse des problèmes et de gestion des situations inhabituelles. À ces grandes évolutions du travail, s'est ajoutée à partir de 2008 une crise économique et financière de très forte ampleur, qui a largement aggravé le chômage des jeunes dans de nombreux pays.

Pour faire face à tous ces changements, le rôle-clé des systèmes de formation professionnelle est à chaque fois souligné, avec le paradoxe que ce secteur éducatif, en particulier au niveau secondaire, reçoit souvent une trop faible considération en comparaison de celle accordée aux cursus généraux. Les réformes préconisées par ces institutions sont largement fondées sur une approche théorique du capital humain que l'on peut synthétiser ainsi: une meilleure éducation se traduit par l'essor de la productivité économique et sociale d'un pays, dans un contexte mondial caractérisé par des économies de plus en plus basées sur la connaissance (Carnoy, 1999). Cela doit donc conduire les états à investir davantage dans leurs systèmes éducatifs pour en améliorer l'accès et la qualité, cette dernière étant entendue comme la production de compétences les plus cohérentes avec les besoins économiques et sociaux actuels et futurs d'une société.

Les transformations préconisées apparaissent largement convergentes dans tous les textes. Elles portent en particulier sur les contenus et les modes de transmission. Les premiers doivent mieux répondre aux évolutions du monde $\mathrm{du}$ travail. Plusieurs rapports soulignent le décalage persistant entre d'une part les savoirs scolaires ou universitaires et d'autre part les besoins des employeurs. Réduire ce décalage nécessite de renforcer les partenariats entre institutions de formation et entreprises pour la définition des curriculums. Sur le plan de la méthode, il faut développer une approche par compétences et la construction de référentiels d'activités, qui doivent ensuite servir de base à la construction des curriculums, via des référentiels de formation et de certification. Ce renfor- 
cement des partenariats ne doit pas être réservé au niveau secondaire, mais être aussi mis en place dans l'enseignement supérieur pour professionnaliser les cursus (Agulhon, Convert, Gugenheim \& Jakubowski, 2012; Stavrou, 2011). Face à l'accélération des transformations du monde du travail, le rythme des révisions de ces référentiels doit s'accélérer. Ainsi, il n'est plus envisageable de faire perdurer les mêmes curriculums pendant huit ou dix ans. Une révision au rythme minimal de tous les trois ou cinq ans est dorénavant nécessaire. Par ailleurs, pour faire face à des situations professionnelles plus imprévisibles et des évolutions de carrière non linéaires, les jeunes doivent être préparés à apprendre à apprendre. Pour ce faire, des compétences plus transversales sont requises et doivent être transmises au cours de leur formation initiale. Il s'agit en particulier de capacités à utiliser les outils numériques, de compétences en communication orale, en littératie (lecture et production d'écrits professionnels), en numératie (mobilisation de savoirs et de techniques mathématiques pour traiter des problèmes professionnels), ou encore en économie ou en sciences.

À travers ces discours, on voit se dessiner l'idée d'un équilibre à trouver entre le développement de compétences spécifiques aux situations de travail et l'acquisition de connaissances plus générales qui reposent assez largement sur des savoirs académiques. Cet équilibre est d'autant plus nécessaire que les formations professionnelles initiales ne doivent pas seulement préparer au monde du travail, mais aussi à une vie citoyenne active, possibilité qui ne doit pas être réservée à une élite éclairée, mais bien concerner tous les jeunes. Enfin, les cursus doivent encore permettre à ceux qui les choisissent, notamment au niveau secondaire, de poursuivre ou reprendre leurs études et d'effectuer facilement des passerelles d'une formation à une autre. La possibilité pour les élèves des formations professionnelles secondaires d'accéder à l'enseignement supérieur est particulièrement soulignée, ce qui nécessite de leur donner les bases théoriques nécessaires. Dans cette perspective, les pays dotés d'un système dual, s'ils sont souvent pris comme modèle par ces institutions pour faire évoluer les formations professionnelles (voir plus loin), sont aussi critiqués pour leur difficulté à favoriser les poursuites d'études dans l'enseignement supérieur.

Sur le plan des modes de transmission, l'importance des phases d'apprentissage en situation de travail ressort unanimement. Le passage suivant, issu d'un rapport de l'OCDE en 2009, est très représentatif de ce que l'on peut trouver dans ces différents discours institutionnels.

Workplace training for young people has compelling advantages. It provides a strong learning environment for both soft and hard skills; it facilitates recruitment by allowing employers and potential employees to get to know each other; it contributes to the output of the training firm; and it links training provision to a direct expression of employer needs. Collectively, these arguments are so powerful that all VET systems should aim to make substantial use of workplace training. (Field et al., 2009, p. 14) 
Il n'est donc plus possible de former les apprenants uniquement dans des écoles ou centres de formation. L'alternance école/travail (sous statut salarié en particulier) apparait comme le modèle à développer dans toutes les formations, y compris au niveau supérieur. Cela souligne à nouveau l'importance de partenariats pédagogiques renforcés entre institutions éducatives et entreprises, cette fois sur le plan d'une division du travail de formation. Mais il ne s'agit pas seulement de plonger des jeunes dans des environnements professionnels. Ces périodes de stage ou d'alternance en situation de travail doivent être bien préparées en amont, bien encadrées pour permettre des apprentissages de qualité, avec en particulier une progression et une variété des tâches en lien avec la formation, et enfin bien exploitées en aval, lors des retours en centre de formation. Plusieurs documents insistent en particulier sur la transférabilité des apprentissages réalisés et recommandent de donner un poids significatif aux évaluations en situation de travail dans l'obtention du diplôme. Les exemples cités sont souvent pris dans les pays disposant d'un système dual ou de filières par alternance très développées (Allemagne, Autriche, Suisse, Finlande, Danemark), qui font figure de modèles pédagogiques.

On peut encore souligner un dernier point caractéristique des différents discours des grandes institutions internationales. À cette logique d'alternance école/travail s'ajoutent des encouragements à l'innovation pédagogique. Aux traditionnels cours en classe et séances pratiques dans les ateliers d'école, s'ajoutent fréquemment des incitations à l'utilisation des nouvelles technologies pour l'enseignement et l'apprentissage. Celles-ci doivent permettre le développement de dispositifs de formation à distance, de simulation de situations réelles, ou encore de logiciels de type jeux sérieux. Les situations d'apprentissage à base de projets ou d'études de cas sont aussi souvent évoquées. Cette diversification pédagogique apparaît comme un gage de modernité, d'attractivité des formations pour les jeunes, et donc de plus grande motivation pour apprendre.

\section{Quelles évolutions des formations dans les pays européens?}

De façon synthétique, on peut donc dire que les discours des grandes organisations internationales préconisent de façon convergente de diversifier et d'enrichir les contenus, et de varier et combiner les modalités de transmission dans tous les domaines et à tous les niveaux de formation, y compris dans l'enseignement supérieur. Mais qu'en est-il du point de vue des évolutions didactiques dans les différents pays? Dans un texte consacré aux réformes récentes des systèmes de formation professionnelle en Europe, Gonon (2008) analyse les évolutions dans différents pays européens, plus particulièrement au niveau secondaire. Cette étude montre que bon nombre des évolutions dégagées ci-dessus sont à l'œuvre dans ces pays. Mais la réception des préconisations se traduit par des actions 
différentes en fonction de l'histoire spécifique de chaque système national de formation.

Ainsi, dans les débats nationaux, la tension historique entre des visées très utilitaristes (former à des besoins immédiats des employeurs) et d'autres plus humanistes (éduquer/cultiver aussi l'Homme ou la Femme et le citoyen) semble aujourd'hui en voie d'être dépassée. Si beaucoup de pays ont longtemps privilégié des savoirs très techniques et pratiques dans les formations professionnelles, en particulier au niveau secondaire, la plupart des décideurs sont maintenant conscients que la préparation stricte à un poste de travail a peu d'intérêt, tant les évolutions économiques, techniques, organisationnelles du monde du travail sont nombreuses et rapides, et finalement assez largement imprévisibles dans leur détail (Agulhon, 2009; Maillard, 2012). Autrement dit, la forte différenciation des filières de formation professionnelle par rapport aux cursus généraux (secondaires ou supérieurs) perd de sa pertinence économique et sociale. Si l'approche par compétences et le principe de construction de référentiels se sont généralisés, y compris dans l'enseignement supérieur (Postiaux, Bouillard \& Romainville, 2010), la construction des curriculums n'est généralement pas seulement basée sur une analyse des pratiques professionnelles existantes, mais inclut bien des références à d'autres savoirs et/ou pratiques issues de disciplines savantes ou d'autres sphères de pratiques culturelles (Durey, 2002). Mais comme les systèmes de formation ont été constitués différemment sur le plan historique, les stratégies pour les faire évoluer ne sont pas identiques.

Les pays ayant mis en place un système de type dual (Allemagne, Autriche, Danemark, Suisse) choisissent plutôt de le consolider par des mesures visant à: 1) développer des compétences transversales en littératie, numératie, ou culture scientifique; 2) faire évoluer les pédagogies utilisées par le biais notamment d'approches basées sur des situations-problèmes permettant d'introduire les savoirs généraux, techniques et pratiques de façon non cloisonnée (Bosch, 2010); 3) élargir ce système dual à certaines formations de l'enseignement supérieur, comme en Allemagne avec la mise en place des Berufsakademies (ou Duale Hochschule), ou tout du moins renforcer la logique d'alternance à ce niveau via des stages plus longs et plus nombreux.

Pour les pays ayant plutôt une tradition de formation professionnelle en école (France, Espagne, Italie, etc.), c'est le renforcement de l'alternance sous statut scolaire (augmentation de la durée et de la fréquence des stages dans les cursus) ou salarié (développement des formations en apprentissage ou d'autres dispositifs équivalents) qui est recherché. Il ne s'agit pas pour autant d'éliminer les temps de formation en atelier d'école, mais de diminuer leur importance horaire au profit des moments d'apprentissage en situation de travail. L'alternance peut aussi s'appliquer à l'enseignement supérieur, comme c'est le cas en France depuis la fin des années quatre-vingt-dix, grâce à l'extension de la possibilité des contrats d'apprentissage à ce niveau. Les réformes peuvent également passer par une action sur les structures des filières, comme c'est encore le cas 
en France avec l'évolution du baccalauréat professionnel vers une formation en trois ans (au lieu de quatre auparavant) en 2009, similaire à la durée des filières générales. Cette réforme visait à développer l'accès au baccalauréat et, partant, aux études supérieures. Dans cette même logique, une stratégie plus radicale encore est de fondre les deux types de filière en une seule, comme en Ecosse et en Suède (Gonon, 2008).

Des recherches menées à des échelles plus microsociales, par le biais de l'étude des dispositifs et/ou des pratiques de formation concrètes, et ce dans des pays disposant de systèmes de formation différents, montrent que ces logiques de complexification didactique semblent effectivement à l'ordre du jour des responsables des filières et des formateurs depuis plusieurs années (Filliettaz, de Saint-Georges \& Duc, 2008; Grubb, 1999; Helle, Tynjälä \& Olkinuora, 2006; Larcher \& Crindal, 2004; Lipp \& Ria, 2012; Pastré, 2011; Perret \& PerretClermont, 2001). Les curriculums apparaissent comme denses en contenus de différentes sortes et riches sur le plan des modalités de transmission. L'inventivité didactique et pédagogique des formateurs de ces filières semble grande. Elle passe par des réflexions régulières sur les meilleures façons de former, dans la recherche d'équilibres ou de compromis entre différentes formes de transmission, anciennes et nouvelles. Dans les écoles ou les centres de formation, à la forme scolaire principalement basée sur le langage oral et écrit dans des classes, et à la forme atelier d'école, historiquement élaborée pour permettre des apprentissages pratiques professionnels, s'en ajoutent aujourd'hui plusieurs autres, comme le recours à des dispositifs de simulation (avec des variations selon le degré de fidélité aux situations réelles), des maquettes didactiques construites par les formateurs eux-mêmes ou des sociétés spécialisées dans ce type de produit, des environnements d'apprentissage en ligne, des situations de projets en petits groupes (conception d'objet technique ou prestation de services pour un client virtuel ou réel), etc. À tout cela, il faut bien entendu ajouter les périodes d'apprentissage en situations de travail de plus en plus fréquentes et/ou longues, encadrées a minima par un tuteur de stage ou d'alternance, et parfois aussi par un tuteur du côté de l'école.

Toutes ces situations se succèdent au cours d'un parcours d'apprenant qui s'avère particulièrement riche en savoirs divers et en formes de transmission et finalement en intervenants de toutes sortes (enseignants disciplinaires, professionnel intervenant de façon occasionnelle au titre de son expertise, formateurs d'atelier, animateurs de projets, tuteurs, etc.).

\section{Les causes sociales potentielles d'un accrois- sement de la division du travail de formation}

Au-delà de la simple réponse des États aux injonctions des grands organismes internationaux, comment comprendre cette tendance à la complexification 
didactique des parcours? Elle semble se traduire par une augmentation de la division du travail de formation entre plusieurs acteurs spécialisés dans des types de contenus ou de modes de transmission. Pour mieux comprendre ce processus, il faut sans doute le replacer dans une évolution plus large des sociétés modernes, dont plusieurs sociologues ont proposé de rendre compte en termes de différenciation sociale croissante des activités et fonctions. Différentes explications théoriques ont été proposées pour comprendre ce processus (Lahire, 2012). Nous en retiendrons quatre, particulièrement intéressantes de notre point de vue pour interpréter les évolutions mises en évidence dans les parties précédentes.

En premier lieu, la différenciation sociale au niveau global d'une société peut être considérée comme la conséquence directe d'une division du travail croissante au sein de la sphère productive. C'est l'hypothèse marxiste (Marx, 1993; Marx \& Engels, 1968) qui considère que la division technique et la division sociale du travail se sont cumulées dans l'histoire, avec un point d'accélération très important depuis la révolution industrielle, pour produire l'extrême variété des fonctions productives actuelles. Et au-delà, sans entrer dans le détail de l'analyse historique et dialectique développée, il y a cette idée fondamentale que les conditions et formes du travail, dans leurs dimensions matérielles et sociales (rapports entre des catégories de travailleurs porteurs d'intérêts différents), produisent les modes d'existence et donc génèrent des effets dans l'ensemble des autres sphères d'activités humaines.

De fait, l'extrême division du travail dans le monde économique actuel a de très grandes conséquences sur les sphères de formation professionnelle. Elle oblige en particulier les créateurs d'une filière à gérer une tension permanente entre une spécialisation de leur formation pour des types d'activités professionnelles très pointues, et une approche plus transversale censée alimenter un ensemble plus large de métiers ou de fonctions entretenant des caractéristiques communes. Une forte spécialisation présente l'avantage de garder un lien formatif très étroit avec un type particulier de métiers et de garantir ainsi un cursus très concret, gage supposé d'une intégration professionnelle rapide et efficace. Mais la multiplication de dispositifs formatifs de ce type a un coût économique et une complexité sociale tels qu'il est très difficile de généraliser cette logique à grande échelle. En effet, plus une formation est spécifique à un petit nombre de métiers, moins il est possible pour des employeurs de domaines d'activités autres d'identifier si les formés sont adaptés à leurs propres spécificités productives. Pour les diplômés, cette situation pose aussi des problèmes importants pour leur mobilité professionnelle, dans un contexte où la perspective de rester dans un seul type de poste tout au long de sa carrière devient de plus en plus improbable (Maillard, 2012).

Une explication différente de la différenciation sociale dans les sociétés, plus politique, est proposée par Elias (2003). À partir d'une analyse très documentée sur le plan historique, il avance que le processus de différenciation dans les sociétés occidentales modernes n'a pu véritablement atteindre un tel degré sans 
que se constitue un double monopole étatique sur la fiscalité et le contrôle de la violence légitime. C'est seulement une fois qu'un État central a pu se mettre en place, après la période du Moyen-Âge, et créer des conditions sécuritaires et économiques plus favorables, notamment par la mise en place d'une administration différenciée (avec différents ministères et services), qu'a pu s'enclencher une différenciation croissante des fonctions sociales dans le reste de la société, dans laquelle l'État a pu jouer un rôle direct, en la favorisant et la régulant volontairement, ou de façon plus indirecte, par les conditions de sécurité et de prévisibilité économique mises en place.

Si l'on reprend ce type d'explication, on voit bien que le monopole fiscal d'un État et son pouvoir législatif lui donnent les moyens d'imposer des règles communes à tous les acteurs œuvrant à la relation emploi-formation. En particulier face à l'extrême différenciation des activités économiques pointées plus haut, les États et leur bureaucratie disposent d'un pouvoir de mise en ordre qu'ils peuvent d'ailleurs en partie déléguer aux partenaires sociaux selon les pays, pour définir un ensemble limité de catégories d'emplois et de filières de formation par des rapprochements, des regroupements et des mises en correspondance. Cependant, il serait trompeur de ne voir qu'une logique limitatrice lors de la construction des formations professionnelles initiales. Car la limitation du nombre de formations à l'échelle nationale se paie de la nécessité de proposer, au sein de ces cursus à plus larges débouchés, un plus vaste ensemble de savoirs si l'on veut pouvoir préparer à une large palette de fonctions ou de métiers au moyen d'une même formation. L'action de l'État et de ses partenaires en matière de formation professionnelle (entreprises, collectivités locales, etc.) serait donc la fois intégratrice, par sa capacité à imposer des filières en nombre limité, avec des règles et procédures bureaucratiques communes, et différenciatrice, par la nécessité de préparer, au sein de chaque cursus, à une large palette de fonctions ou métiers, en y incluant aussi de plus en plus des visées de préparation culturelle plus générale qui dépassent la stricte logique professionnelle.

Or ces deux processus se sont sans doute accentués depuis quelques années sous le poids de deux facteurs. Tout d'abord, il est bien évident que d'importantes considérations financières entrent en ligne de compte dans ces interventions des pouvoirs publics, d'autant plus lorsque la conjoncture économique est mauvaise. Celles-ci poussent à une réduction du nombre de formations pour des raisons de coût et à privilégier les formations qui proposent un plus grand nombre de débouchés et donc un contenu formatif plus large. Par ailleurs, l'influence des organismes supranationaux (OCDE, UE, UNESCO, BIT, etc.), qui ancrent leurs discours dans la théorie du capital humain, porte à former mieux et plus longtemps un public plus large de jeunes et donc plus diversifié socialement. Cette norme des études longues tend d'ailleurs à s'imposer de plus en plus dans les esprits des jeunes et de leur famille dans beaucoup de pays, y compris dans ceux qui disposent d'un système dual. Ces influences encouragent les pouvoirs publics nationaux à réorganiser leur système de formation, en redéfinissant des 
objectifs de qualification à différent niveaux (secondaire court et long; supérieur court et long) et en créant des possibilités de passerelles d'un niveau ou d'un domaine à un autre. Les nouveaux publics accueillis (plus diversifiés), ainsi que l'exigence de mobilité accrue d'une formation à l'autre, conduisent là aussi à introduire des contenus nouveaux et à différencier les situations et approches pédagogiques pour mieux gérer cette hétérogénéité nouvelle.

Rosa (2010), un autre sociologue allemand, développe de son côté une réflexion sociologique sur les évolutions du rapport au temps au sein de nos sociétés modernes. À partir de différentes sources empiriques et théoriques, il met en évidence l'accélération des changements techniques, économiques, organisationnels et culturels au sein de nos sociétés modernes. Une telle accélération, dont la cause est à trouver dans différents facteurs, n'a pu être mise en œuvre qu'au prix d'une différenciation très nette de sphères de pratiques de plus en plus spécialisées et optimisées sur le plan temporel. En particulier, il semble bien que le processus historique de séparation des pratiques de formation de celles du monde du travail ait été très important pour l'émergence d'une pensée et de pratiques pédagogiques centrées sur la recherche des voies les plus adaptées pour préparer les jeunes aux sphères professionnelles (Veillard, 2017). La séparation des temps formatifs et productifs a permis de se dégager des contraintes économiques et donc temporelles propres au monde du travail et d'ouvrir un vaste périmètre de réflexions sur ces questions pédagogiques et didactiques. La différence des rythmes temporels propres à ces deux types de sphères est très nette et de nombreuses situations de formation mises en œuvre dans les écoles semblent de fait incompatibles avec le temps très fortement contraint en vigueur sur les lieux de production. Mais dans le même temps, si ces différences de rythmes s'accroissent de façon trop importante, elles constituent un risque fort pour la fonctionnalité et donc la crédibilité des systèmes formatifs. Face à des sphères professionnelles dont les transformations des pratiques, des techniques et des organisations n'ont cessé de s'accélérer, en particulier depuis une quarantaine d'années, les sphères de formation se sont vues sommées de réagir. Or, il paraissait difficile d'accélérer les processus de formation dans la mesure où, simultanément, les exigences en termes de complexité des compétences professionnelles n'ont fait que s'accentuer. Il y a donc une contradiction importante entre le caractère de plus en plus éphémère, mais néanmoins de plus en plus complexes, de nombreuses pratiques professionnelles et le temps nécessaire pour y préparer solidement les jeunes.

Comment résoudre cette équation apparemment insoluble? On a vu que la réponse de nombreux prescripteurs et décideurs en matière de formation professionnelle consiste à mettre en place une synchronisation temporelle beaucoup plus étroite entre les organisations productives et formatives. Plusieurs dispositifs sont proposés pour le permettre: se rapprocher des milieux professionnels pour construire conjointement les curriculums au sein de commissions se réunissant de plus en plus fréquemment; solliciter davantage des intervenants profes- 
sionnels pour assurer des cours ou des parties de cours; renforcer le rôle des périodes d'apprentissage en situation de travail, via des stages ou mieux encore, par une organisation de la formation en alternance; tisser des partenariats avec des milieux professionnels pour la fourniture d'équipements; mettre en place des projets réalisés par les élèves et encadrés par des enseignants en réponse à des demandes émanant d'employeurs, etc. Tous ces dispositifs convergent: tisser un réseau d'interdépendances toujours plus étroites entre les sphères de formation et les sphères de production pour assurer un arrimage temporel plus prononcé entre elles. Mais ceci n'est pas sans poser d'importantes questions, comme celle de changements de curriculums trop fréquents qui ne permettent plus aux formateurs de stabiliser leurs pratiques, ou encore celle du risque de désynchronisation temporelle en interne entre les différentes parties d'une formation. Sur ce dernier point, on peut facilement imaginer qu'un décalage croissant de rythme puisse se créer entre des enseignements de matières générales, dans lesquels les contenus et les méthodes de transmission sont relativement stables, et des cours professionnels qui sont sans cesse soumis à des changements de programmes et à un renouvellement fréquent des modes de transmission, pour mieux correspondre aux mutations professionnelles. À la différenciation des contenus et méthodes d'enseignement dans ces différents contextes disciplinaires, s'ajouterait donc une différenciation des rythmes temporels qui semble de fait bien réel, quand on compare les rythmes des activités proposées dans les différents cours (Veillard, 2015).

Lahire (2012) rappelle qu'un quatrième type d'explication a été proposé en s'appuyant sur le travail de Goody (1979), relatif au rôle de la littératie dans la différenciation des pratiques sociales au sein des sociétés humaines. L'écriture constitue une technologie cognitive très importante qui permet de développer une plus grande réflexivité sur les spécificités des pratiques, sur les savoirs liés ou produits par celles-ci, en même temps qu'ils participent objectivement de leur spécialisation et séparation. C'est ainsi qu'ont pu se créer et se différencier du reste des autres activités humaines, des pratiques savantes autonomes, orientées vers l'étude ou l'organisation de différents domaines du réel matériel et social. À partir du $19^{\mathrm{e}}$ siècle, cette logique de différenciation des pratiques de production des connaissances selon des disciplines toujours plus pointues s'est très fortement accélérée, avec à la clé une professionnalisation de leurs acteurs. En reprenant cette idée pour analyser les évolutions des sphères de formation, l'écriture apparaît comme un instrument central de la construction des curriculums actuels, lors des étapes de la création des référentiels d'activité puis de formation notamment. C'est par des jeux d'écriture que sont menées les opérations réflexives de repérage ou de création des similarités de certaines pratiques professionnelles dans les commissions de construction des référentiels. Celles-ci aboutissent à des descriptions écrites unificatrices, mais généralement sous une forme standardisée, beaucoup plus abstraites et imprécises que les pratiques réelles en situation (Caillaud et al., 2012; Darré, 1994; Raisky, 1995). En ce 
sens, le langage contribue à limiter les effets de la différenciation propre aux sphères professionnelles sur les sphères de formation.

À l'inverse, lors de l'étape suivante de création du référentiel de formation, les concepteurs doivent également décider quels sont les différents contenus de savoir permettant de préparer aux métiers et tâches formalisés précédemment. Là aussi le rôle des écrits est décisif, toujours en lien avec les conséquences de la différenciation sociale des activités humaines. Mais il ne s'agit plus d'unifier des pratiques diverses en domaines structurés. À l'inverse, l'objectif est d'éclater la description unitaire réalisée en de multiples textes de savoir disciplinaires ou modulaires qui feront l'objet d'un enseignement séparé. Cette séparation s'impose pour au moins deux raisons: d'une part, des raisons historiques qui ont conduit les établissements d'enseignement professionnel à reprendre l'organisation disciplinaire en vigueur dans l'enseignement général secondaire et supérieur (Chervel, 1988); d'autre part, des raisons liées à une certaine conception hiérarchique des rapports entre les savoirs explicatifs théoriques et les pratiques professionnelles qui s'est imposée dans nos sociétés occidentales (Schön, 1983) et demeure encore profondément ancrée, malgré un affaiblissement certain de cette façon de penser (Stavrou, 2008). On peut la formuler ainsi: si l'on connait les principes de fonctionnement des matériels, méthodes de travail et organisations, alors on est capable d'en inférer des solutions pratiques pour résoudre les problèmes posés dans tout type de situation professionnelle. Les jeux d'écriture qui sont réalisés visent à produire des mises en correspondance entre des pratiques professionnelles et des savoirs issus d'autres sphères (savantes ou culturelles). Ces mises en correspondance n'ont souvent rien de naturel, mais ont un effet indéniable, en contribuant puissamment à la création et légitimation des différents discours de savoirs qui serviront de référence aux enseignants pour mettre en place les différentes situations d'enseignement (Delbos \& Jorion, 1984).

\section{Quelques conséquences didactiques de la division du travail de formation}

Pour terminer, tentons maintenant de cerner les impacts de ces évolutions sur les curriculums des apprenants. Concrètement, la division accrue du travail de formation se traduit pour eux par un plus grand nombre de contextes d'apprentissage tout au long de leur cursus. On entend ici par contexte d'apprentissage, un dispositif mis en place pour assurer la transmission d'un certain type ou groupe de savoirs, et généralement conçu et/ou animé par un type d'intervenant(s): cours assuré par un enseignant d'une discipline, animateur encadrant un module de projet, tuteur entreprise encadrant un stagiaire, etc. Des contextes d'apprentissage plus nombreux ont deux conséquences didactiques importantes.

Tout d'abord, ils requièrent de la part des apprenants une souplesse accrue pour s'adapter à des changements plus ou moins subtils de contenus de savoirs, 
de rapports à ces contenus (étude d'une notion en physique ou utilisation de cette notion comme concept outil pour une tâche technique), de rapport aux objets (compréhension du fonctionnement de l'appareil ou apprentissage de son usage), de configurations de l'espace, d'organisation sociale (travail seul, en dyade, en groupe), d'attentes de l'enseignant ou enseignante en termes d'action, de comportement, de façon de s'exprimer, etc. (Veillard \& Lambert, 2015). Ces nombreuses transitions d'un contexte à un autre, qui mettent donc en jeu toute une série de ruptures, mais aussi parfois de continuités, sont assez peu questionnées par les responsables et formateurs. Pourtant, on peut s'interroger sur la capacité de tous les apprenants à bien cerner les attentes et spécificités propres à chaque contexte, en particulier ceux qui ont un parcours plus difficile sur le plan scolaire et peuvent davantage peiner à cerner les nombreux implicites en jeu dans ces transitions (Bonnéry, 2007; Jellab, 2008). On peut aussi se demander s'ils sont toujours en capacité de comprendre en quoi chaque enseignement ou situation d'apprentissage contribue à leur formation professionnelle plus globale.

Une seconde difficulté est de parvenir à relier les apprentissages réalisés dans les différents contextes d'apprentissage et les intégrer pour construire les compétences complexes attendues en fin de formation. L'éclatement modulaire ou disciplinaire, s'il est abondamment utilisé dans les univers formatifs, est totalement inadéquat en situation de travail, comme le montrent de nombreux travaux sur les compétences: celles-ci apparaissent comme des systèmes de connaissances très intégrées, structurées pour l'efficacité et l'efficience de l'action dans un type de situation (Le Boterf, 2008; Pastré \& Samurçay, 2001). La plupart du temps, l'apprenant doit parvenir à faire seul ce travail de mise en lien et d'intégration. Plusieurs auteurs ont souligné le caractère très problématique de cette organisation des connaissances dans les lieux de formation (Bransford \& Schwartz, 2001; Eraut, 2004; Pastré, 2008), car elle freine grandement les possibilités de transfert des connaissances entre les différents contextes formatifs. Le problème n'est certes pas nouveau, mais l'accroissement de la division du travail de formation le renforce. Pour essayer de dépasser ce type de difficultés, des propositions ont été faites pour développer des curriculums plus intégrés.

Beaucoup préconisent de rompre avec la division disciplinaire des enseignements et de réorganiser les curriculums autour de cas pratiques ou de situations-problèmes typiques d'un métier. On retrouve ce type de propositions en particulier dans certains rapports de grandes organisations examinés précédemment. Cela est censé introduire progressivement toutes les connaissances nécessaires à l'étude de ces situations, puis à leur gestion ou résolution dans un même contexte d'apprentissage. L'idée apparaît séduisante pour développer des compétences plus proches de celles attendues en situation de travail. Mais elle pose question dans un contexte de différenciation sociale croissante au sein de nos sociétés. En effet, si l'on considère que les jeunes seront amenés à évoluer dans des univers sociaux composés de multiples sphères d'activité spécialisées, 
et devront donc être capables de circuler relativement aisément entre elles (les activités de type projet sont typiques de cette souplesse sociale), les capacités à s'adapter à des attentes et fonctionnements sociaux, économiques ou techniques différents, ainsi que l'aptitude à réaliser des transfert de connaissances ou de techniques d'un contexte à un autre, deviendront de plus en plus déterminantes, en particulier pour générer de nouvelles idées, concevoir des produits ou services innovants (Engeström, Engeström \& Kärkkäinen, 1995). Il y a donc un enjeu très important à ce que les cursus de formation y préparent les jeunes: qu'ils soient capables, par exemple, de faire des liens entre des organisations spécialisées dans la production de connaissances (laboratoires de recherche ou de R\&D) et d'autres tournées vers la production de biens et services (entreprises). Ce n'est sans doute pas en mettant en place des curriculums toujours plus proches des situations de travail que l'on y parviendra le mieux.

Une voie sans doute plus intéressante consiste à prendre acte de la nécessité d'une diversité de contextes d'apprentissage (tout en maintenant la division du travail de formation dans des limites raisonnables), mais à se préoccuper bien davantage de l'accompagnement des apprenants lors des transitions entre ces différents contextes. La différenciation au sein des cursus de formation semble inévitable dans la mesure où différents types de connaissances doivent être transmis dans les formations et qu'il est très difficile de le faire avec les mêmes types de dispositifs didactiques (uniquement des situations-problèmes par exemple). Ainsi, bien apprendre des notions en physique nécessite une approche et des moyens matériels et symboliques particuliers que l'on ne trouvera pas dans un atelier d'école, ou lors d'un stage en entreprise. Mais encore faut-il que les apprenants aient compris pourquoi tel ou tel concept en physique (par exemple: les changements d'état de la matière) est important dans leur cursus, pourquoi il est nécessaire de passer par un détour disciplinaire (le cours de physique) dans un type d'espace particulier (le laboratoire de physique) pour apprendre ce concept, puis enfin comment les notions vues lors de ce cours peuvent ensuite être transférées et adaptées dans d'autres contextes pour aider à la compréhension du fonctionnement d'un objet technique (une climatisation). Cette voie est aujourd'hui travaillée par quelques chercheurs, souvent en collaboration étroite avec des formateurs (Senström \& Tynjälä, 2009; Tuomi-Grohn \& Engeström, 2003; Veillard \& Kouamé Kouassi, 2014). Des propositions sont faites pour développer des pédagogies plus connectives ou intégratives, par exemple en mettant en place des activités de mise en lien entre différentes sphères de pratiques (boundary-crossing activities). Toutes ces propositions mettent l'accent sur l'importance du travail collaboratif entre les différents formateurs d'un cursus (enseignants des disciplines générales, formateurs d'atelier, tuteurs en situation de travail) et parfois même aussi avec les apprenants. Il y a là indéniablement un axe stratégique majeur pour faire évoluer les pratiques au sein des formations professionnelles initiales qui restent encore sans doute beaucoup trop individuelles. 


\section{Bibliographie}

Agulhon, C. (2009). Les relations entre formations et emplois: une adéquation improbable. Formation emploi, 107, 73-76.

Agulhon, C., Convert, B., Gugenheim, F. \& Jakubowski, S. (2012). La professionnalisation. Pour une université utile? Paris: L'Harmattan.

Billett, S. (2011). Vocational education. Purposes, traditions and prospects. New York: Springer.

Bonnéry, S. (2007). Comprendre l'échec scolaire. Elèves en difficultés et dispositifs pédagogiques. Paris: La Dispute.

Bosch, G. (2010). The revitalisation of the dual system of vocational training in Germany. In G. Bosch \& J. Charest (Éd.), Vocational training. International perspectives (pp. 136-161). New-York, NY: Routledge.

Bransford, J. D. \& Schwartz, D. L. (2001). Rethinking transfer: A simple proposal with multiple implications. Review of research in education, 3, 1-42.

Caillaud, P., Gosseaume, V., Garrigues, R., Grumeau, C., Labarrade, G., Kalck, P. \& Paddeu, J. (2012). Place et rôle des professionnels dans la conception des diplômes professionnels (Net.doc, 89). Marseille-Cedex: Céreq.

Carnoy, M. (1999). Mondialisation et réforme de l'éducation: ce que les planificateurs doivent savoir. Paris: UNESCO.

Chervel, A. (1988). L'histoire des disciplines scolaires. Réflexions sur un domaine de recherche. Histoire de l'éducation, 38, 59-119.

Darré, J. P. (1994). Les voies de construction d'un référentiel: le cas des conseillers techniques agricoles. In F. Rope \& L. Tanguy (Éd.), Savoirs et compétences. De l'usage de ces notions dans l'école et l'entreprise (pp. 147-174). Paris: L'Harmattan.

Delbos, G. \& Jorion, P. (1984). La transmission des savoirs. Paris: Maison des Sciences de l'Homme.

Durey, A. (2002). Impact de la CAO sur la définition des contenus d'enseignement en génie électronique. In J. Lebeaume \& Y. Cartonnet (Éd.), Séminaire de didactique des disciplines technologiques, 2001-2002. Bilan des recherches en didactique des disciplines technologiques (pp. 7-29). Cachan: Association Tour 123.

Elias, N. (2003). La dynamique de l'occident. Paris: Pocket.

Engeström, Y., Engeström, R. \& Kärkkäinen, Y. (1995). Polycontextuality and boundary crossing in expert cognition: Learning and problem solving in complex work activities. Learning and instruction, 4, (5), 319-336.

Eraut, M. (2004). Transfer of knowledge between education and workplace settings. In H. Rainbird, A. Fuller \& A. Munro (Éd.), Workplace learning in context (pp. 201-221). London: Routledge.

Evans, D. (2007). The history of technical education: A short introduction (2e éd.). Cambridge: T Mag Extra.

Field, S., Hoeckel, K., Kis, V. \& Kuczera, M. (2009). Learning for jobs OECD. Review of vocational education and training (Initial Report). Paris: OCDE.

Filliettaz, L., de Saint-Georges, I. \& Duc, B. (2008). «Mais vous tapez comme un pharmacien!” Des analogies en formation professionnelle initiale. In L. Filliettaz \& M.-L. SchubauerLeoni (Éd.), Processus interactionnels et situations éducatives (pp. 137-158). Bruxelles: De Boeck.

Gonon, P. (2008). Apprenticeship, vocationalism and opposing VET-Reform trends in Europe. In V. Aarkrog \& C. H. Jorgensen (Éd.), Divergence and convergence in education and work (pp. 57-76). Bern: Peter Lang.

Goody, J. (1979). La raison graphique. La domestication de la pensée sauvage. Paris: Minuit.

Greinert, W.-D. (2004). European vocational training systems. Somes thoughts on the theoretical context of their historical development. European journal of vocational training, $32,18-31$. 
Greinert, W.-D. (2005). Mass vocational education and training in Europe. Classical models of the 19th century and training in England, France and Germany during the first half of the 20th. Luxembourg: CEDEFOP, Office for Official Publications of the European Communities.

Grubb, W. N. (Éd.). (1999). Honored but invisible. An inside look at teaching in Community Colleges. London: Routledge.

Helle, L., Tynjälä, P. \& Olkinuora, E. (2006). Project-based learning in post-secondary education: Theory, practice and rubber sling shots. Higher education, 51, (2), 287-314.

Hyslop-Margison, E. (2000). An assessment of the historical arguments in vocational education reform. Journal of career and technical education, 17, (1), 23-30.

ILO. (2010). Global dialogue forum on vocational education and training (Final report). Geneva: ILO.

Jellab, A. (2008). Sociologie du lycée professionnel. L'expérience des élèves et des enseignants dans une institution en mutation. Toulouse: Presses Universitaires du Mirail.

Lahire, B. (2012). Monde pluriel. Penser l'unité des sciences sociales. Paris: Le Seuil.

Larcher, C. \& Crindal, A. (2004). Nouveaux dispositifs, nouvelles rencontres avec les connaissances. Aster, 39, 3-9.

Le Boterf, G. (2008). Repenser la compétence: pour dépasser les idées reçues: 15 propositions. Paris: Éditions d'Organisation.

Lipp, A. \& Ria, L. (2012). La transmission des savoirs en formation professionnelle initiale: Analyse de l'activité d'enseignants en lycées agricoles. Activités, 9, (2), 71-87.

Maillard, F. (2012). Professionnaliser les diplômes et certifier tous les individus: une stratégie française indiscutable? Carrefours de l'éducation, 34, (2), 29-44.

Marx, K. (1993). Le capital. Tome 1. Paris: Presses Universitaires de France.

Marx, K. \& Engels, F. (1968). L'idéologie allemande. Paris: Editions Sociales.

OCDE. (2015). Perspectives de l'OCDE sur les compétences 2015: Les jeunes, les compétences et l'employabilité. Paris: Editions de l'OCDE. doi :10.1787/ 9789264235465-fr

Pastré, P. (2008). Apprentissage et activité. In Y. Lenoir \& P. Pastré (Éd.), Didactique professionnelle et didactiques disciplinaires en débat (pp. 53-79). Toulouse: Octarès.

Pastré, P. (2011). La didactique professionnelle. Paris: Presses Universitaires de France.

Pastré, P. \& Samurçay, R. (2001). Travail et compétences: un point de vue de didacticien. In J. Leplat \& M. de Montmollin (Éd.), Les compétences en ergonomie. Toulouse: Octarès.

Pelpel, P. \& Troger, V. (1993). Histoire de l'enseignement technique. Paris: Hachette.

Perret, J.-F. \& Perret-Clermont, A.-N. (2001). Apprendre un métier dans un contexte de mutations technologiques. Fribourg, Suisse: Éditions Universitaires.

Postiaux, N., Bouillard, P. \& Romainville, M. (2010). Référentiels de compétences à l'université. Usages, rôles et limites. Recherche et formation, 64, 15-30.

Raisky, C. (1995). Référentiel professionnel: quitter les évidences trompeuses. In Actes du colloque "Territoires ruraux et formation» (pp. 377-387). Dijon: ENESAD.

Rosa, H. (2010). Accélération. Une critique sociale du temps. Paris: La Découverte.

Schleicher, A. (2006). The economics of knowledge: Why education is key for Europe success. Brussel: The Lisbon Council asbl.

Schön, D. A. (1983). The reflective practitioner. New York, NY: Basic Books.

Senström, M.-L. \& Tynjälä, P. (2009). Towards integration of work and learning. Strategy for connectivity and transformation. New York, NY: Springer.

Stavrou, S. (2008). La recontextualisation à l'épreuve de la sociologie empirique des curriculums: éléments de recherche sur la «régionalisation du savoir.» In D. Frandji \& P. Vitale (Éd.), Actualités de Basile Bernstein (pp. 171-187). Rennes: Presses Universitaires de Rennes.

Stavrou, S. (2011). La "professionnalisation» comme catégorie de réforme à l'université en France: de l'expertise aux effets curriculaires. Cahiers de la recherche sur l'éducation et les savoirs, 3, 93-109. 
The World Bank. (2002). Constructing knowledge societies: New challenges for tertiary éducation. Washington, DC: World Bank.

Tuomi-Grohn, T. \& Engeström, Y. (2003). Conceptualizing transfer: From standard notions to developmental perspectives. In T. Tuomi-Grohn \& Y. Engeström (Éd.), Between school and work: New perspectives on transfer and boundary crossing (pp. 20-37). Oxford: Elsevier.

UE. (2010). The Bruges Communique on enhanced cooperation in Vocational Education and Training for the period 2011-2020. European Union. Retrieved from http://ec.europa.eu/ dgs/education_culture/repository/education/policy/vocational-policy/doc/brugescom_ en.pdf

UNESCO-UNEVOC. (2015). UNEVOC TVET strategy 2016-2021. Report of the UNESCOUNEVOC virtual conference (vol. 1). Bonn : UNESCO-UNEVOC.

Veillard, L. (2017). La formation professionnelle initiale. Apprendre dans l'alternance entre différents, Rennes: PUR.

Veillard, L. \& Kouamé Kouassi, D. (2014). Conception d'un outil d'aide à la mobilisation de connaissances enseignées en formation par alternance. Éducation et francophonie, 13, (1), 42-64.

Veillard, L. \& Lambert, P. (2015). Analyse comparée des processus didactiques dans trois contextes d'apprentissage en lycée professionnel. In F. Chnane-Davin \& J.-P. Cucq (Éd.), Approche comparative des savoirs et des compétences en didactiques (pp. 277-298). Paris: Riveneuve.

Mots-clés: Formation professionnelle initiale, organisations internationales, réformes éducatives, différenciation sociale, curriculum d'apprentissage

\section{Die Aufteilung in der beruflichen Grundbildung: soziale Gründe und didaktische Konsequenzen}

\section{Zusammenfassung}

Seit den industriellen und politischen Revolutionen haben viele Staaten Berufsausbildungssysteme mit spezifischen Strukturen entwickelt. In diesem Artikel untersuchen wir den Diskurs grosser internationaler Organisationen, die den Staaten empfehlen, Reformen auf der Grundlage gemeinsamer didaktischer Prinzipien durchzuführen. Der Text stützt sich weiter auf vier soziologische Theorien der Differenzierung und erfasst die tiefgreifenden sozialen Gründe, die zu einer zunehmenden Arbeitsteilung in den Ausbildungen der verschiedenen Ausbildungsrichtungen führen. Schliesslich untersuchen wir die Auswirkungen dieser Veränderungen auf die Ausbildungswege der Lernenden.

Schlagworte: Berufliche Erstausbildung und Weiterbildung, internationale Organisationen, Erziehungsreformen, soziale Differenzierung, Lehrplan 


\title{
La divisione del lavoro di formazione nelle formazioni professionali iniziali: cause sociali e conseguenze didattiche
}

\section{Riassunto}

In seguito alle rivoluzioni industriali e politiche, numerosi Stati nazionali hanno sviluppato dei dispositivi specifici di formazione professionale iniziale. Tuttavia, con il fenomeno della globalizzazione, sembra delinearsi una maggiore convergenza dei sistemi nazionali verso alcuni principi didattici comuni. Il presente articolo analizza come il discorso delle organizzazioni internazionali spinge gli Stati nazionali a intraprendere delle riforme in tal senso e, di conseguenza, a modificare le realtà curricolari locali. In seguito, l'articolo si propone di rilevare le cause sociali profonde che generano una crescente divisione del lavoro nelle diverse filiere di formazione. Infine, l'articolo considera le conseguenze di tali cambiamenti sui percorsi formativi degli studenti.

Parole chiave: Formazione professionale iniziale, organizzazioni internazionali, riforme educative, differenziazione sociale, curriculum di apprendimento

\section{The division of training labour in initial vocational education and training Systems: Social causes and educational consequences}

\begin{abstract}
Since the period of industrial and political revolutions, numerous countries have built Vocational Education and Training (VET) Systems with strong national specificities. In this paper we analyse several institutional discourses delivered by major international organisations, which advocate for vocational curricula evolutions around common pedagogical principles. Several reforms have been done in each country in accordance with these recommendations. As we demonstrate further, theories about sociological differentiation show deeper social reasons, which can explain why countries change their VET systems towards an increasing division of the training labour in a lot of vocational courses. Finally, we discuss some important consequences, generated by these transformations, for the students' learning paths.
\end{abstract}

Keywords: Initial vocational education and training, worldwide organisations, education reforms, social differentiation, learning curriculum 